\title{
SUL, REGIME PLUVIOMETRICO DI FIRENZE (1832-1949)
}

\author{
Regination Cianferoni
}

T,Osiervatorio meteorologieo del Musco dell'Cniversila di Firenze " possierle una serie ininlerrotta di osservazioni della quantità e della frefuenza delle precipitazioni dal 1832 arl ogri. L’intensità della piogrgia in invere, revistrata da tempo relativamente recente. In questa nota terremo conto solo degli elementi dei quali si possierlono i ralori completi per l'intero periorlo. Arrertiamo, però, che i rati degli anni 1867. 1868. 7869, 1870, 1871. 1872. 1873 e 1933, 1934, 1935, 1936 sono guelli raceolti dall'Oservatorio Ximeniano fposto nella stessa cilla di Firenze a cirra 1200 metri di distanza), perché, in quegli ami, gli strumenti di raceolta di letto Osservatorio non polerono funzionare in modo sicuramente attendibile.

Le medie rle verranno fornite nel eorso di questa nota, qualehe volta. si diseostano da quelle calcolate o rese note nell'" Anmuario del

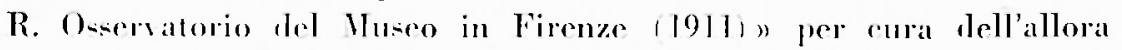
direttore Antonino to Surlo. Cii i dovuto alla circostanza sopra accennala e al periorlo più lungo di osservazioni ora posserlule.

Ta piongria e la neve carlute sono raceolte in un unieo dato: la neve i registrata in mu secondo la quantita di acqua proveniente dalla slla fusione.

Quantità media della proggia mei 718 anmi $11832-19491$ "scostamenti massimi, - Ún cosi lungo periodo di anni di osservazioni può permetlere di ricercare se vi sono state variazioni nelle carateristiche di questo elemento neleorolognico.

Infalti prentendo in esame. non l’intero periorlo di 118 anmi. ma frazioni piì o meno lunghe di tale periorlo si nota che le relative medie sono diverse. e qualche volta notevolmente, le une dalle altre. Ciis non acrarle fer altri elementi meteorologici. i quali. pur arendo

1*1 Coordinate geografiche: latit. $13^{0}+6^{\prime}-$ longit. $1^{\prime \prime} 12^{\prime} \mathbb{W}-$ altitudine sul livello del mate m. 50. 
una variabilita ammua piuttosto grande ripetono, in un numero sulficientemente lungo di amni. le stesse medie. Per questultimo latto si può escludere che si tratti di un'oseillazione climatiea a periodo più o meno lunge perehe in lal case satrelole diflede immaninare che tale oscillazione non influenzasse anche altri clementi meteorologici. Ad uguale conclusione, del restos si grunge esaminambe i dati plusiome-

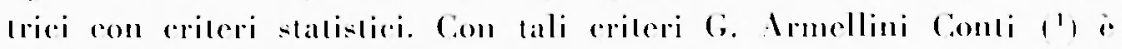
grumta, infatti, alla conelusione ehe le variazoni notate di anno in anno nella quantita di piogegia sono interpertabili come mo fenomeno catilate.

Tuttavia is molto prohalbile che le medic alcolate per lintero feriodo varieranmo di poco anche quando vi saramo altri numerosi anni di osservazioni a meno che - süntende - non si registrino reali variazioni elimatiche che possono essere dovute anche allepera dell'nomo trimboschimenti o diboseamenti ecel. Per i 118 anmi considerati. la meelia annuale della piogrgia i di mm 8388.8 15.6. 1.o seostamento medio di ogni anno risuha di - $170 \mathrm{~mm}$. Entro tale scostamento medio sono compresi 89 dei 118 anni. mentre i rimancnti 29 ne sono fuori ed assumono quindi un rarattere di ecerzionalita.

Gli scostamenti piò forti in meno, rispetlo alla media anmuale, si somo registrati: nel 189.1 in eni caddero mon 399.0 di piogyia: nel

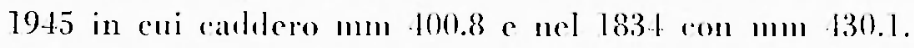

D'altra palte gli scostamenti più forti in più si somo aruli nel 1838 con piogerial di mon 13.19.9: nel 1851 con mm 1157.l: nel 1898 con mu 11.4 .7 e nel 1815 con mm 11.13.4.

Distribuzione mensile della quamità. -. Per remelere facile il confronto fra il regime pluviometrico della localita presa in esame e quello deggli altri luoghi e gria usata ta molto tempo in meteorolog̣ia la media relativa, overo la media espressa in millesimi della media ammat media mensile $x$ mille $)$ media ammua

Tultavia questo metodo, se rente confrontabili fra di loro i diversi regimi pluviometrici. non rende ugualmente confrontabili fra di loro i singِoli mesi a causa del diverso numero di piorni ali cui sono

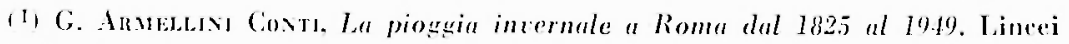
Fend. Sc. fis. mal. e nat., vol. $1 \mathrm{I}$, gingno 19.19. 
composti. Per ragriungere invece, contemporancamente, anche quest'ultimo scopo ahliamo moltiplicato la sopraseritla formula per $\frac{365}{g \times 12}$ in cui g $\mathrm{e}$ il numero dei riorni del mese. Per semplicita febbraio è stato consitlerato di 28 giomi. Lil media relatira risulta così perequata dal numero dei goiorni del mese. Chiameremo la prima Mr e la secondal Mrt).

\section{TABELLA I}

Medie - Medie relative - Wedie rolative perequale? - Estromi massimi " minimi di riosscan mose

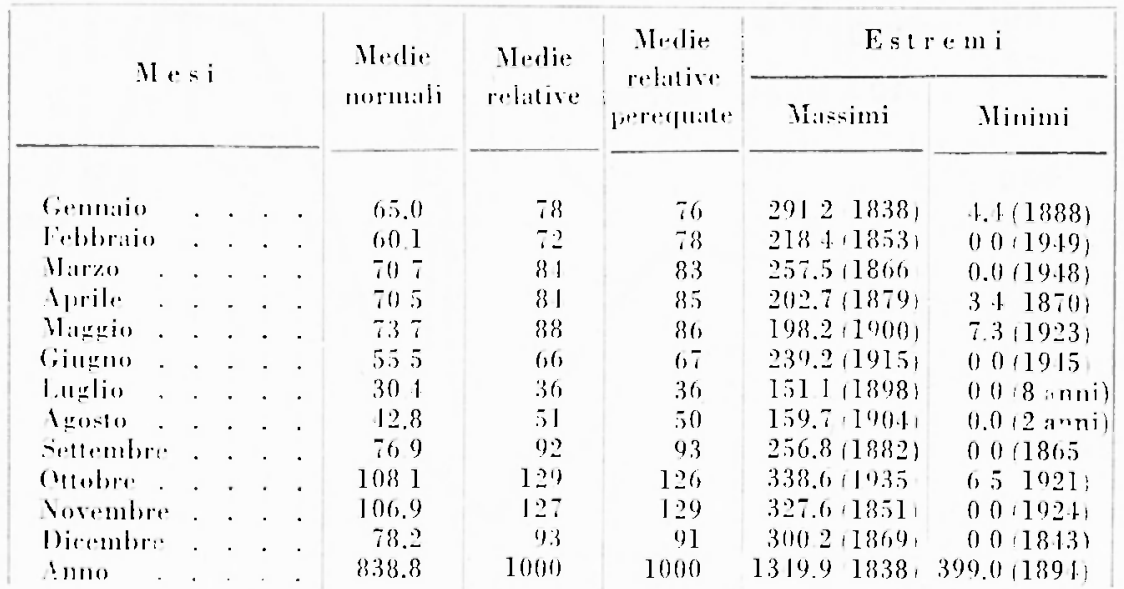

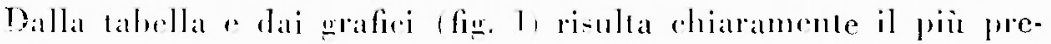
ciso significato della media relativa perequata nei confronti delle medie comunemente adotlate: mentre il gralieo ricarato dalle medie del mese. o da quelle relative presenta delle irregolarita fal esempio fobbraio ha un minimo secondarion in quello ricavato dalla media relativa perequala la curva is piu regolare fat esempio. anziché presentare oscillazioni in più e in meno. wennaio. febbraio, marzo, aprile. malquio salquno qradualmente. Il massimo secondo la Mrp si ha in novembre anziche in ottohre.

Con le sole medie mensili. stazionali o anmali is diffeile determinare il regime pluviometrico di ma localita. Infatti tali medie possomo exsere il risultato di distribuzioni molto diveros nelle quantiti di precipitazione. Allo seopo di illustrare queste distribuzioni per 


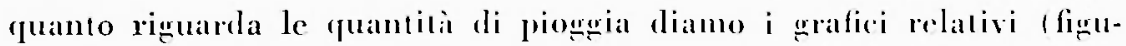
re 2 -a e $2-1$ ).

Come risulta dai gralici $\mathrm{i}$ mesi con valori inferiori alla media sono sempre in numero maggiore di quelli con valori superiori. Nei mesi le frequence massime delle altezze di precipitazione si hanno sempre a valori inferiori alla media. Cio - con maggriore evidenza si vede per fehlumio, giugno. lughlio, ollohre. Nel loro complesso i mesi che lanno valori inferiori allat loro media sono il $57 \%$.

Per quanto riguarda l'attendibilita delle medie mensili formite,

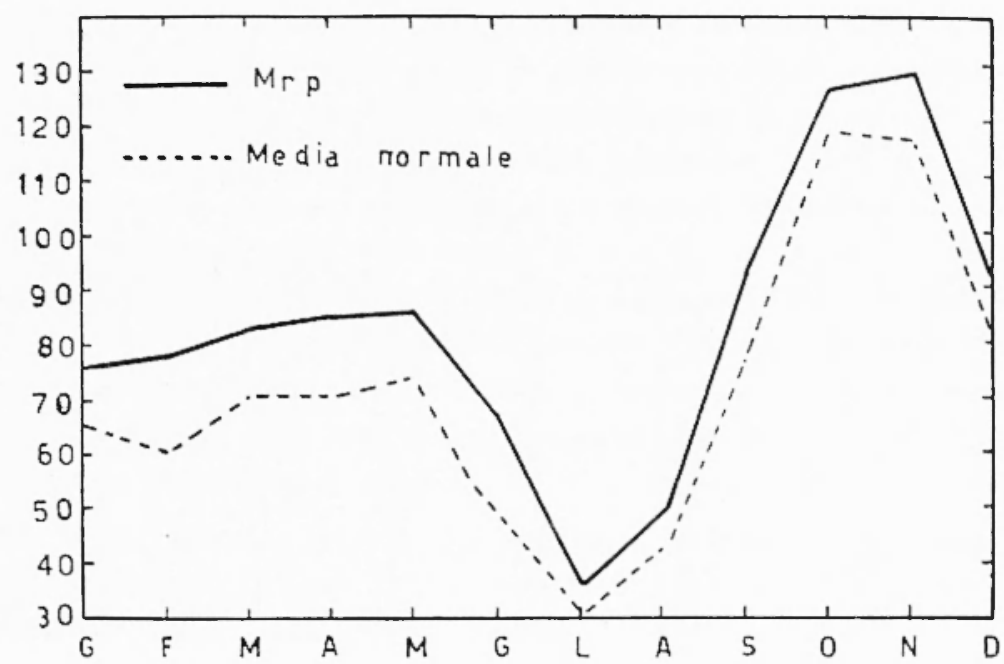

Fif. l Valori medi mensili della cpuantiti della precipitazione

e allo scopo di dare un'indicazione, diamo per grennaio lo seostamento della media: 4. Lo scostamento medio i di $\cdots 47$. Ventotto volte, nel corso dei 118 anni, i mesi di gennaio presentano scostamenti maggriori.

Distribuzione stagionale della quantitì. - Supponendo che le precipitazioni siano, nella loro quantita, ripartite uniformenente durante tutti i mesi dell'anno e facendo le dillerenze con la piogrgia che effettivamente carle ricaviamo la tahella II. Nella tabella II sono pure riportate le medie e grli estremi stagionali. 


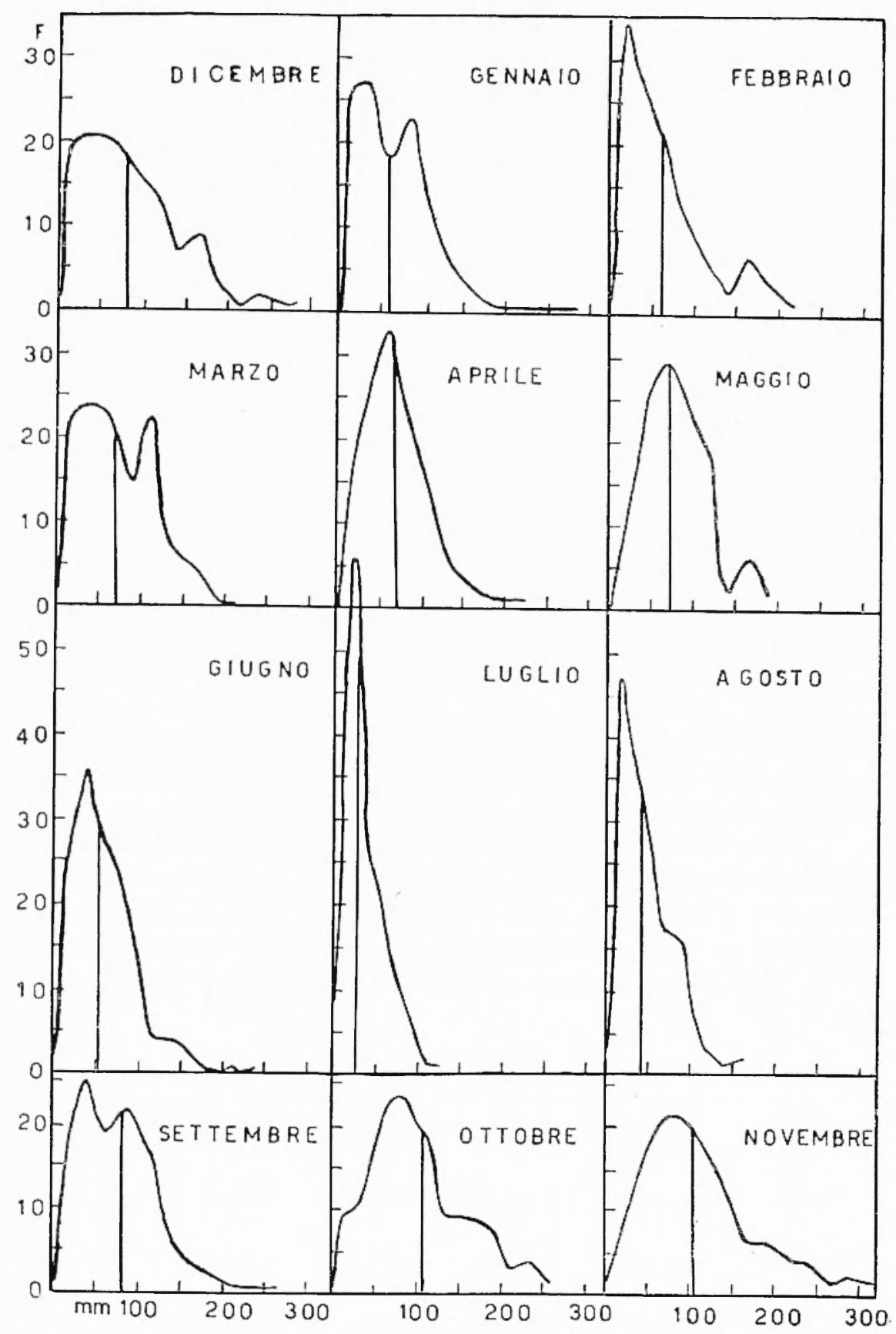

Fig. 2a) - Frequenze mensili della quantità di precipitazione 
TABELLA II

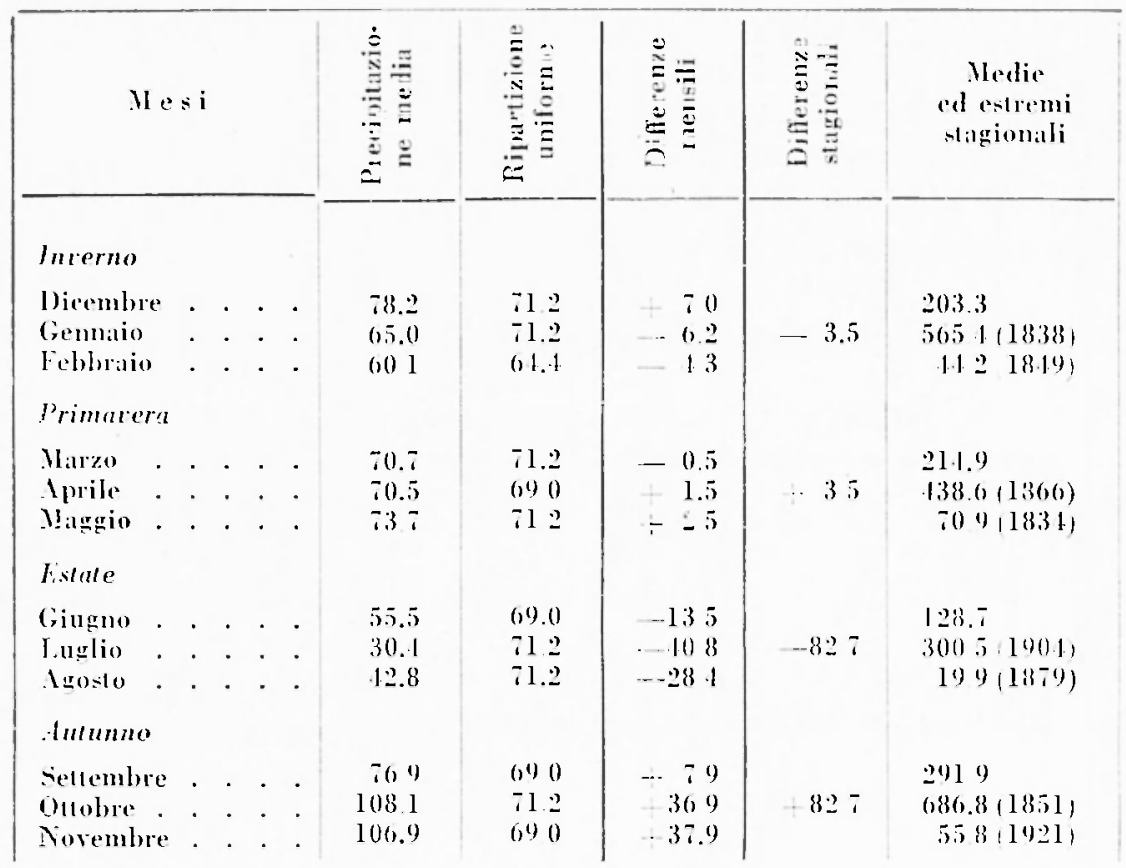

Abbiamo dunque - anche se la coincidenzal dei numeri i puramente casuale - equesto fenomeno aratteristico: la piowwial che viene in meno d'estate viene in piì d'autumno.

L'andamento plusiometrieo stagionale si pui anche illustrare contando il numero delle volte in cui i i verificata una certal suecessione delle quattro stagioni (I inverno. $P$ primavera, $E$ estate. $I$ autumos classificate in ordine decrescente secondo la quantiti di piowgial I risultati, nelle ventiquattro permutazioni posibitili sono riportate nella tabella III.

\section{TABliLta III}

\begin{tabular}{|c|c|c|c|c|c|}
\hline $\begin{array}{c}\text { M.I.I.E. } \\
21\end{array}$ & $\begin{array}{l}\text { A.I.P.E. } \\
18\end{array}$ & 1.P.E.I. & A.I.F.I'. & $\underset{I}{\text { A.E.I.I. }}$ & $\begin{array}{c}\text { A.I.I.I' } \\
2\end{array}$ \\
\hline $\begin{array}{c}\text { P.A.I.E. } \\
7\end{array}$ & ${ }_{i}^{\text {P.A.E. }}$ & P.I. I.E. & I'E.I.A. & $\begin{array}{c}\text { P.I.E. } \\
\text { I }\end{array}$ & P.1E 1.1. \\
\hline $\begin{array}{c}\text { I.A.P.E. } \\
9\end{array}$ & $\begin{array}{c}\text { I.P.A.E. } \\
7\end{array}$ & I.A.E.I. & $\begin{array}{c}\text { I.P.E.A. } \\
3\end{array}$ & $\underset{3}{\text { T.F.A.P. }}$ & I.E.I. \\
\hline $\begin{array}{c}\text { E.A.P.I. } \\
1\end{array}$ & E.P.I.I. & $\underset{0}{\text { E.I.I.I' }}$ & E.P.I. & FIII.A. & 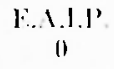 \\
\hline
\end{tabular}




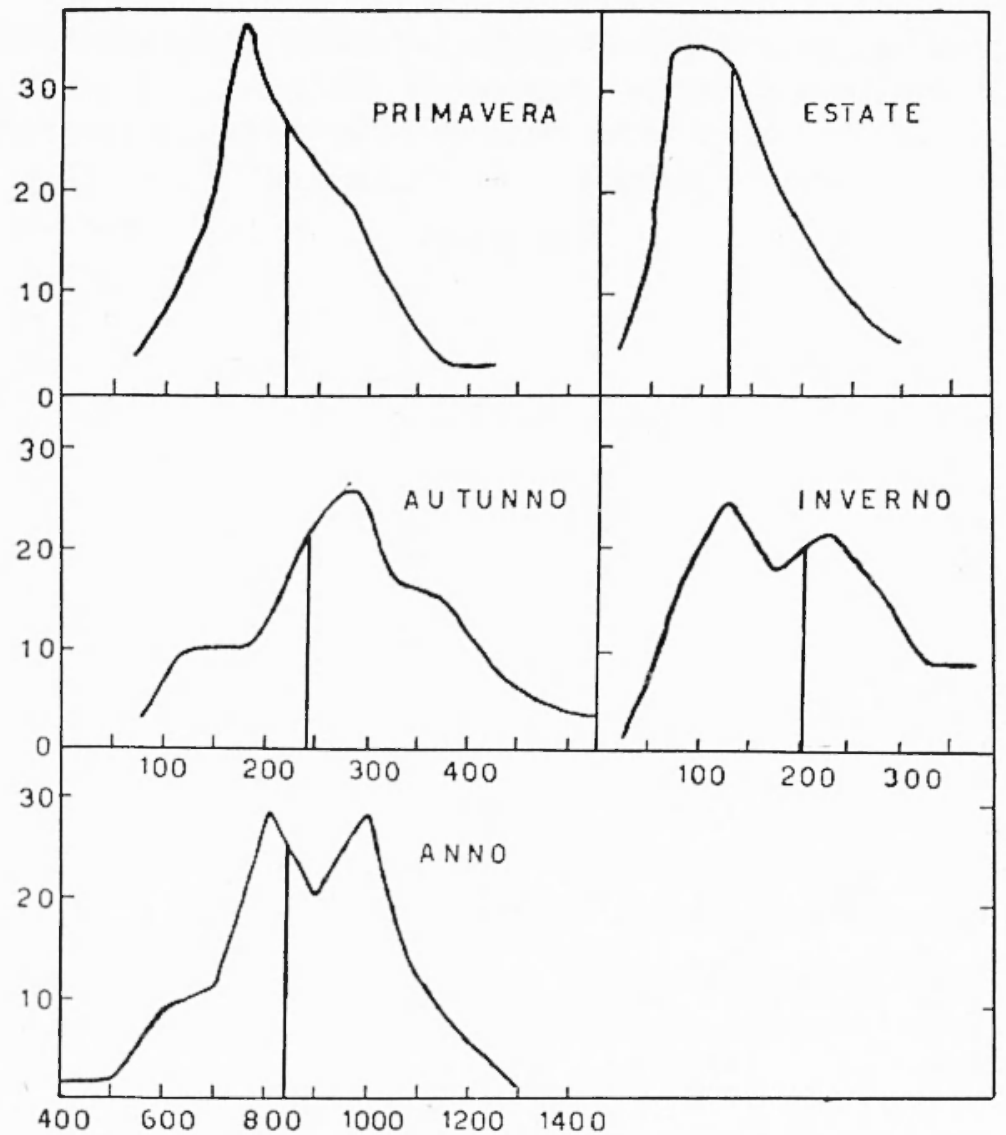

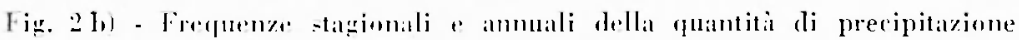

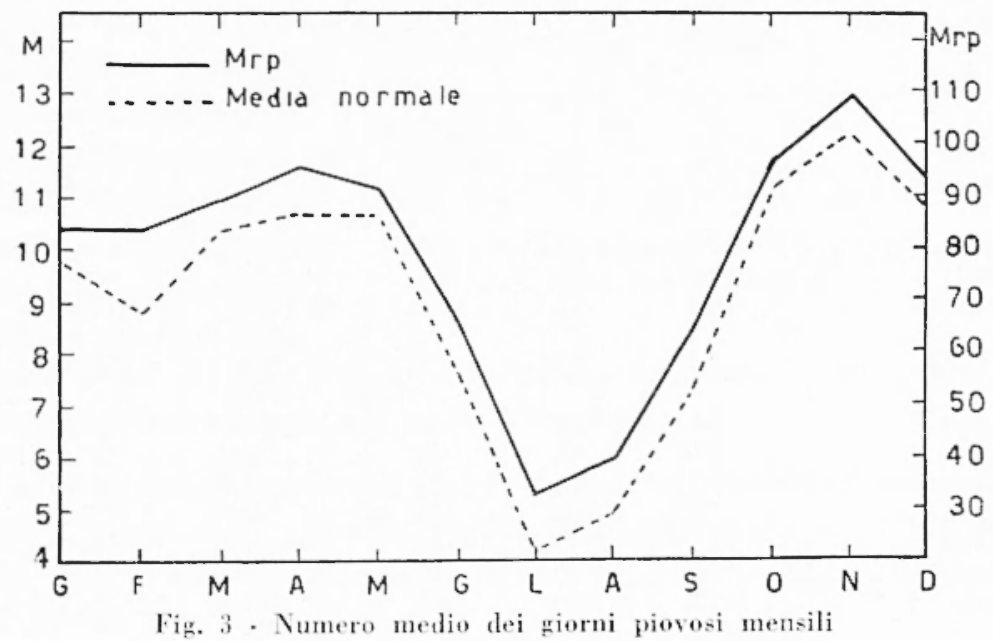


I'ordine stagionale delle medie freavabile dalla tabella IIj i quello che ha la frequenzal maggiore $(2 \mathrm{l}$ voltel.

Le singole stagioni hanno oceupato nella suecessione quantitativa delle precipitazioni i scguenti posti:

TABELIA IV

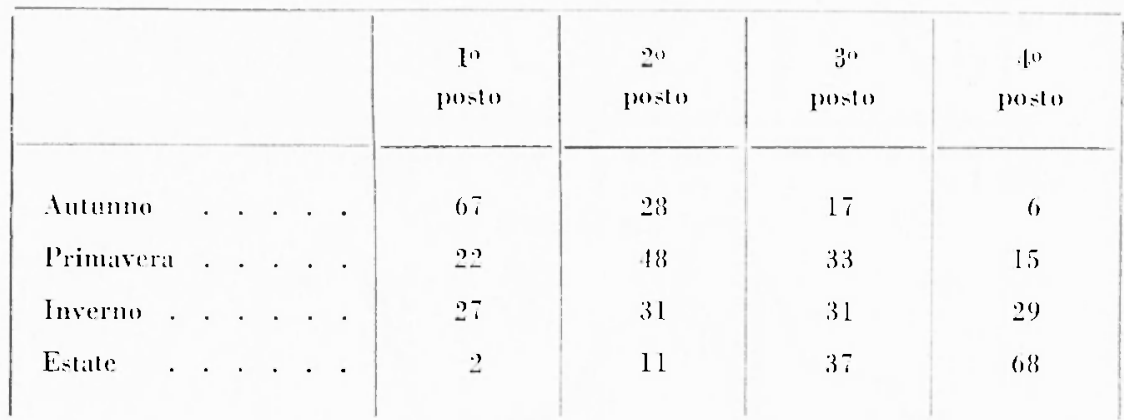

Risulta. dunque confermato l'opposto regime dell'estate e dell'autunno: l'inverno e la primavera -.... che secondo le medie sono stagioni poco diverse -- si diflerenziano un po' perche wli inverni sono distribuiti quasi ugualmente nei quattro posti, mentre la primatreral si trova preferibilmente nei due posti centrali.

Sudio della jrequenzu. - La media amuale dei giorni nei quali si è avuta una precipitazione misurabile o di lo7.8. Il massimo sco-

TABILLA V

\begin{tabular}{|c|c|c|c|c|c|c|c|}
\hline \multirow{2}{*}{\multicolumn{3}{|c|}{ Descrizione }} & \multirow{2}{*}{$\begin{array}{l}\text { Merlie } \\
\text { normali }\end{array}$} & \multirow{2}{*}{$\begin{array}{l}\text { Medie } \\
\text { relative }\end{array}$} & \multirow{2}{*}{$\begin{array}{c}\text { Medie } \\
\text { relative } \\
\text { londerate }\end{array}$} & \multicolumn{2}{|c|}{$\mathrm{E}=\mathrm{tr} \mathrm{rmi}$} \\
\hline & & & & & & $\mathrm{II}_{\mathrm{a}-\mathrm{i}: \mathrm{int}}$ & Minimi \\
\hline Genuaio & . & . & 9.7 & 90 & 88 & $20,1856)$ & 218061 \\
\hline Flbbrais & . & . & $8 . \overline{7}$ & 81 & 88 & $\begin{array}{r}21 \\
(1853) \\
(1879)\end{array}$ & $0(19.19)$ \\
\hline Marzo & . & & 10.3 & 96 & 9.4 & 21.18888 & $0: 19.18$ \\
\hline Aprile. & & & 10.6 & 98 & 100 & $24.1879)$ & 1. (1865) \\
\hline Magrio & & & 10.6 & 98 & 96 & $\begin{array}{c}20,185.51 \\
11851\end{array}$ & $1(1923)$ \\
\hline Giumno & & & 7.6 & $\succsim 1$ & $\pi 1$ & $\begin{array}{r}16,1881 \\
\quad(1909\end{array}$ & $1(1836$ \\
\hline Luglio & & & 4.2 & 39 & 38 & $12(1932)$ & $0(8: m n)$ \\
\hline Agosto. & & & 4.9 & 45 & 4. & $17(1896)$ & $0(1832 \div 1915)$ \\
\hline Seltemire & & • & 7.3 & 68 & 69 & $20(1882)$ & $0(1865)$ \\
\hline Otoblere & & & 11.1 & 103 & 101 & $21 \quad 1889$ & $1|1921|$ \\
\hline Novembre & & & 12.1 & 112 & 114 & $20,1878)$ & 01926 \\
\hline Dicembre & . & . & 10.7 & 99 & 97 & $2.1(1869)$ & $0(1843)$ \\
\hline Anno & . & . & 107.8 & 1000 & 1000 & $157(1853)$ & $52(1945)$ \\
\hline
\end{tabular}


stamento da questa media in senso positivo si i verificato nel 1853 con 157 gionmi piorosi. Il masimo scostamento in senso negatio nel 19.15 an giomi 52.

Ancle per la freforenza delle precipitazioni albliamo quincli una grande variabilita me si puic velere dai Elateri lfiw. !).

La talrella Y riporta le medie mensili ol ammuali dri giomi ron precipilizione.

1 caural dellat dirersa intensita modia per owni giorno pioso- a della piogeia mensile l'ordine di suecessione dej mesi seremblo i) numero delle precipilarioni is alisereo da quella della quamlita.

Questat diversita is lun malratat dallat laleclla VI nellal quales taumo fere le lrepluenze romfromli analoghi a quelli fatui per le fualuliti alla tal,. II. Risultal rhe inverno. primatrera ed autumo fresentano tulli un numero di precepitazioni steperiore a quello che si arrabe con la ripar-

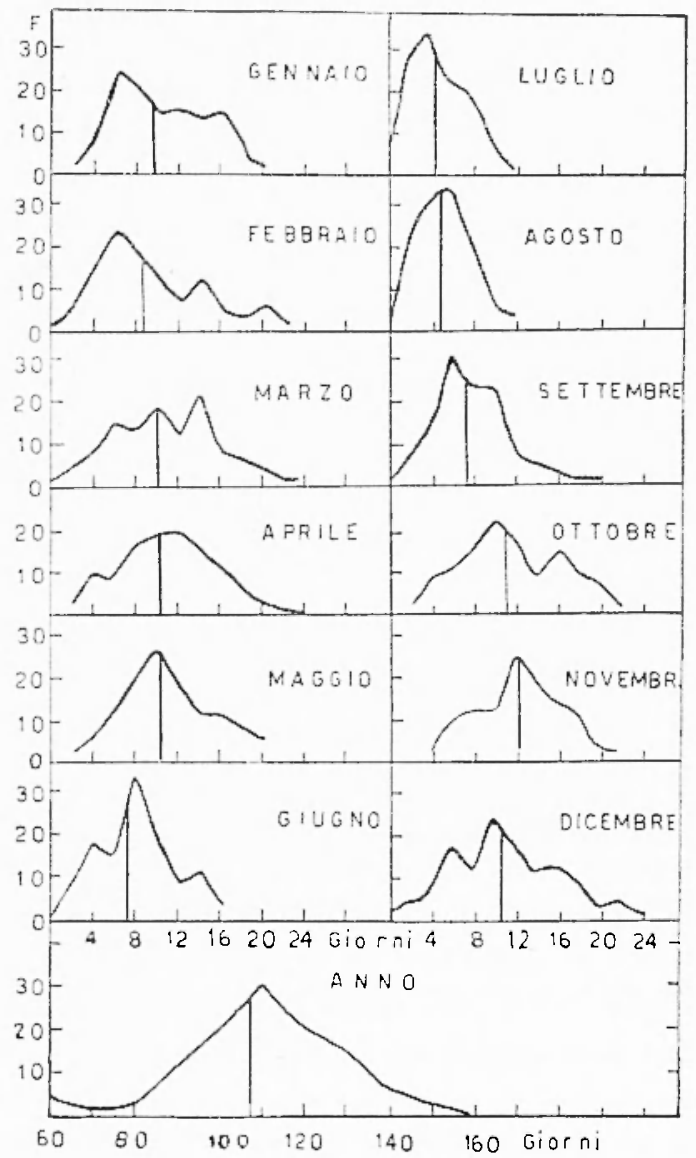

Fig. I - Ferefuenze mensili e annuali dei giorni ron precipitazione lizione uniforme delle preciptazioni durante lamno. L'estate ha in meno i gromi con precipitazione che sono in più nelle altre stạioni.

Intensiti medict per ogni grorno piocoso di ciascun mese. - Dividendo i valori della quantita media di piogegia per i corrispondenti valori della freduenza otteniamo la intensita media della piogria per ogni griorno pioroso. 


\section{TABHLLA VI}

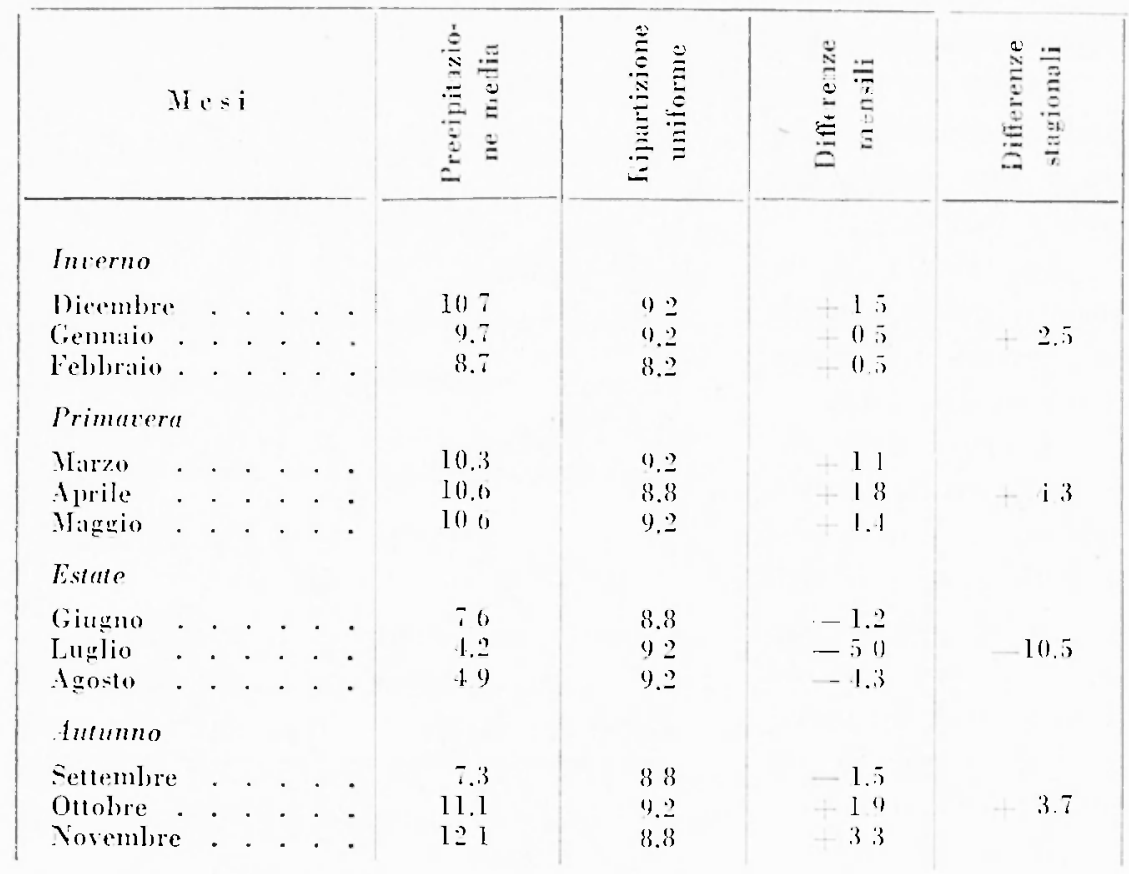

Nella tahella VIl indichiamo lali valori e yli cotremi minimi e massini verificatisi in ciascun mese e nei sinnoli anni.

\section{TABela III}

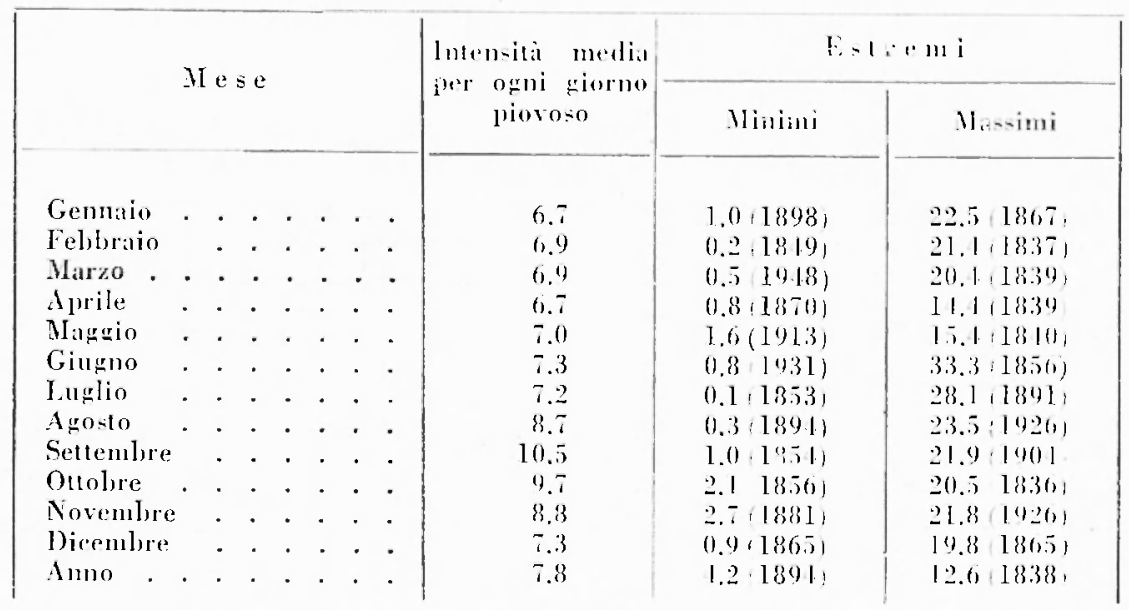


is ahiaro che le precipitazioni minime e massime assolute annuali (tabrella l) coincidono - - come dimostra la tabella VII - con i minimi e i masimi dell’intensila media per ogni griorno piovoso. F precisamente l'amno 189 l regristro la minima quantita assolula di piogrgia frerhe i 95 geromi con precipitazione, che sono di poco inferiori alla merlia, chbero la minima intensita media ali pioggia; l'amo 1838 repi-Lri la masima precipitazione ammale perehe elhe la massima inlensita di proggiat. In quell'anno, infatti, i giomi piovosi (106) furono vicinisimi alla media $1070 \% .81$.

Da cio -i puo dedurre clue l’intensila media della piogreia - per la meno a Firenze - ha ma grande influenza nel determinare la fuantita totale anmuale delle precipitazioni. Naturalmente anche il mumero dei giorni pionosi ha. in tal senso, la sua influenza e, infatti, l'anmo 1915 che chbe il minimo dei giomi piovosi (52) registro mm 400.8 di precipitazioni: mentre l'anno 1853 che ebbe il massimo dei

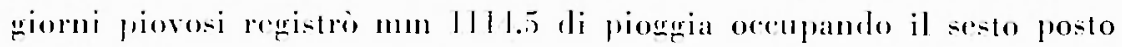
nella graduatoria dei mas-simi.

Erentuale corrolazione con l'altivilì solare. - La ricerea di una erentuale influenza dellattivila solate sulle preciphiazioni i, cerlamente: molon difleile perehe la piogeia. come dimostrano i lati ripartati precedentemente. ha un arattere molto vario e casuale.

Per inve-tigare man eventuale correlazione con i fenomeni solari sarehbe mecesario poter isolare i fattori particolari ehe, senza dublio, -omo yuedli che detorminano lat grande variabilita delle precipitazioni e. per questo. aceorremehte disporre di dati di un vastiszimo territorio o. menlio. dell’inera superficie della terra in modo che le fluttuazioni locali si compensis-sero fra di loro. Questo non 2 per il momento possibile: tultavia si potrehle pensare che il gran numero di anni a nostra disposizione ci permetta di avere - con il fattore tempo anziché con quello -pizio -..- una sufficiente se non completa, compensazione tanlo da poter tratre alcune indicazioni che phr avendo hisogno di più sicure conferme, potreblero servige di hase per altre fulure riererelie.

Yon a salo posibile fienoscere alema correlazione dal confronlo fra la pioggria calula rlurante il periodo di ascesa dell'attivita solare e quella caduta durante il periodo di discesa di tale attiviti. Cis era preverlibile se leventuale influenza viene altribuita all'entita merlia lelle perturtrazioni solitri poiche in lal cato in ambedue le fasi als- 
hiamo una somma di perturbazioni press'a poeo nowale. Quello ehe varia $\dot{e}$ solo la latitudine media delle macehie.

Ho allora messo a conlronto in ciasem ciclo fualtro anni altorno al massimo di attivita solare coi quattro anni di attivita solare minima. omettendo grli altri. Fal cero il risulato esposto nella tabella VIll.

\section{TABIILILA VIII}

\begin{tabular}{|c|c|c|c|c|}
\hline \multicolumn{2}{|c|}{$\begin{array}{c}\text { Ami di minima allivilì } \\
\text { solare }\end{array}$} & \multicolumn{2}{|c|}{$\begin{array}{c}\text { Anni di missimal altivilit } \\
\text { solatre }\end{array}$} & \multirow{2}{*}{ 31:ax-?1m } \\
\hline Anni & $\begin{array}{l}\text { P'iogrgia } \\
\text { dei tanni }\end{array}$ & Anni & $\begin{array}{l}\text { Piogngia } \\
\text { dei l anni }\end{array}$ & \\
\hline 18321835 & 2757.9 & 1836.1839 & 1.329 .0 & $15 i 1$ \\
\hline $18+1 \quad 18+4$ & 3115.8 & $1816-18.49$ & 33.1 .4 & $\begin{array}{r}209.1 \\
+\end{array}$ \\
\hline $185 \% 185 \%$ & 3155.0 & $1858-1801$ & $27+1.5$ & -1103 \\
\hline $186.5-1868$ & 3551.5 & $1809 \cdot 1872$ & $34: 211$ & 20100 \\
\hline 18761879 & $325+.5$ & $1881-1881$ & 29114 & -313.1 \\
\hline 1887.1890 & 3072.8 & $1892-1895$ & 3155.2 & 821 \\
\hline $1899 \quad 1902$ & 3717.2 & 190.11907 & 3736.6 & 19.8 \\
\hline 1911.1911 & 3117.1 & 1915.1918 & 33.15 .1 & $\therefore \quad 1930$ \\
\hline $1921-192.4$ & 28.1 .2 & $102019 \cdot 20$ & $326: 0$ & 126.1 \\
\hline $1931-1934$ & 390.1 .7 & $1936-1939$ & 3373.3 & - -531.1 \\
\hline $19.12-19.15$ & 2772.1 & 1916.19 .49 & 33352 & $\begin{array}{r}50.3 .1 \\
+\end{array}$ \\
\hline 44 anmi & 35323.1 & & $3737+2$ & 2051.1 \\
\hline Media annuale & 802.8 & & 819.1 & +15.7 \\
\hline
\end{tabular}

Negli 11 eonfronti della tabella VIII abhiamo dungue whe per otto volte é piovuto di piit nei quattro anni di masimo e meno nei quattro anni di minimo. Per tre volte si is avuto il catoo inverso.

La piogria totale degli anni di minimo risulta - nei confronti degli amni di massimo - pari al 94.5\%.

La media degcli 11 valori nell'ultima colonna della tabella VIII risulta $+187 \mathrm{~mm}$ (piog̣gia in più dei quall ro amni di masima nei confronti dei quattro anni di minima attivita solares con un errore medio di $161 \mathrm{~mm}$. Come si vede la supposta influenza dell'attivita solare è dubbia anche a causa del forte seostancente registrato nei primi due quardrienni.

E necessario anche notare che il fenomeno notato non si riproduce considerando i mesi anziché gli anni. Infalli prendendo i mesi con $R<1.0$, in cui $R$ ì il numero di Wolf, i nesi cioè nei quali l'attivita delle macchie era duasi inesistente a che, quindi, dovrebbero avere al massimo grado la notata infuenza, questi non presentano nessun comportamento particolare. Il che perio potreble voler dire 
soltanto rle l'influenza lelle macehic solari sulla pioggria non è immediata.

Per un controllo circa l'attendibilita regli scostamenti riportati nella tabella VIll ci posiamo servire anche dello scostamento medio ammale che precedentemente abbiamo trovato essere uguale a +170

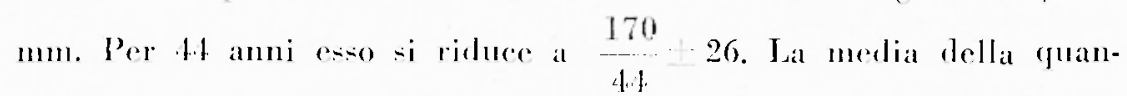
liti di pioggia rartuta attomo al minimo dell'attivià solare va un po' oltre tale scostamento risultamdo guesto - 36. La media della quantita di piogena caduta negli anni di ma-sima attivita solare, invece, rientra nei limiti di tale seostamento risultando questo di $10 \mathrm{~mm}$. Tullavia questi fatti risultano ulteriomente confemati - per brevita non riportiamo i dati relativi -- prendendo, anziché quattro, tre anni intorno al minimo e al massimo attivitit solare.

In conclusione pero. é molta incerteza su una eventuale correlazione fra l'atlivita solare e il regime pluviometrico di Firenze e (ii) era da aspettarsi per le ragioni areconnate. Molto cautamente si pui acennare rome durante gli anni di minima allivita solare si verifihereblero condizioni farorevoli ar ma minore piovosia fatto che risulta ron margriore evidenza dalle ricerche sulla crescila anmule de⿻li alberi secolari ed anche da altri analoghi sturli.

Mi i grato dovere ringraziare il prof. Giorgio Abetti e il prof. M. G. Fracatoro per i suegerimenti datimi.

Osservatorio Astrofisice di Arcelri osservatorio Meleorologico del Museo di Firenze - Marzo 1950.

\section{RI ISSUNTO}

Si damno le carafleristiche del regime placiometrico di Firenze riguardanti la quantilit ola frequenza delle presipitazioni. seconde $i$ dati racolli dal $18 \% 2$ al $70 . \%$.

Si studiano eventuali corrolazioni fra lablivita solare o il regime piariomelrien di Firenze. 


\section{BIBIIOGRAFIA}

(1) trchitio meteorologico centrale italiano nell I. L. R. Museo di Fisica Storia Naturale. Firenze 1858.

(2) Annali del R. Museo di Fision e Soria Naturale di Firenze per il 1865. Nuova Serie, rol. I, 1866.

(3) Pubblicazioni periodiche di meteorologia a cura dellossereatorio del Museo: $18: 2.190 \%$.

(4) A. lo Suroo, Anumario del R. Osservalorio del Maseo in Firenze (1911). Firenze 1911.

(ग) G. Alfavi, Ln secolo di ossertazioni meteorologiche. Firenze 1921). 